Check for updates
Cite this: Nanoscale Adv., 2019, 1, 4119

\section{Analysis of nonlinear characteristics of a graphene based four-terminal ballistic rectifier using a drift- diffusion model}

\author{
Ankur Garg, (D) ${ }^{a}$ Neelu Jain, ${ }^{a}$ Sanjeev Kumar, ${ }^{\text {b }}$ Shahrir R. Kasjoo ${ }^{c}$ \\ and Arun Kumar Singh (D) *a
}

\begin{abstract}
In this study, rectification behavior and noise spectra of a graphene based four-terminal ballistic rectifier are reported utilizing semi-classical drift-diffusion 3D modeling. The room temperature DC and RF characteristics of the novel rectifier are demonstrated considering the traps in the material similar to a real device, reducing the rectification efficiency from $0.5 \%$ to $0.35 \%$. The responsivity and noise equivalent power of about $89.21 \mathrm{mV} \mathrm{mW}^{-1}$ and $97.52 \mathrm{pW} \mathrm{Hz}^{-1 / 2}$, respectively, are obtained for different frequencies varying from $50 \mathrm{~Hz}$ to $1 \mathrm{THz}$. Furthermore, the noise spectral analysis of the device predicts a minimum low frequency noise, which depends upon the carrier concentration inside the device active region rather than mobility, and hence enables potential applications as $\mathrm{THz}$ detectors for imaging.
\end{abstract}

Received 31st December 2018 Accepted 8th September 2019

DOI: $10.1039 / c 8 n a 00423 d$

rsc.li/nanoscale-advances

band gaps to make it operational; hence it is favorable to use single layer graphene as an active layer. The planar structure and in-plane contact configuration generate less parasitic capacitance which enhances the speed of the FTBR up to THz frequencies. ${ }^{16} \mathrm{~A}$ triangular antidot intentionally placed in the center of the FTBR provides asymmetry to the device structure. Furthermore, the antidot deflects a large number of carriers toward the lower terminal and is responsible for the rectification. Previously, these devices have been fabricated using 2DEG materials such as GaAs and InGaAs to work up to a frequency of $50 \mathrm{GHz}$ experimentally ${ }^{17}$ and have predicted the operation up to THz frequencies using Monte-Carlo (MC) simulations. ${ }^{6}$ Other devices such as artificial nanomaterials ${ }^{18}$ and TTJ $^{9-11}$ have also been reported to generate a rectified output. The BDT, which is the modification of the FTBR, is demonstrated to perform digital operations including a NAND gate. ${ }^{8,19}$ The nonlinear response of the FTBR can be explained using an extended Landauer-Büttiker approach, where transmission probability of charge carriers and the electrochemical potential difference between terminals are considered for predicting rectified DC outputs. ${ }^{20}$ In addition, the billiard ball transport model has been used strategically for the deflection of charge carriers toward output terminals using the triangular antidot. ${ }^{20,21}$ The potential applications of the FTBR include energy harvesting, ${ }^{5}$ frequency doubling, ${ }^{22}$ voltage inversion, and THz imaging. ${ }^{23}$

Single layer graphene can be transferred on different substrates such as $\mathrm{SiO}_{2}$ and $\mathrm{h}-\mathrm{BN}$ by exfoliation. The substrate used is responsible for different graphene properties including mobility and hence $\lambda_{\mathrm{MFL}}$. For the first time, graphene FTBR fabrication was reported using a $\mathrm{SiO}_{2}$ substrate with the mobilities of 1572 and $1872 \mathrm{~cm}^{2} \mathrm{~V}^{-1} \mathrm{~s}^{-1}$ for holes and electrons, respectively. ${ }^{24} \mathrm{Here}$, the $\mathrm{SiO}_{2}$ substrate provided a rough surface 
to graphene which created puddles and was responsible for the lower mobility. Recently, the graphene FTBR is fabricated on the h-BN substrate providing higher mobility $\left(200000 \mathrm{~cm}^{2} \mathrm{~V}^{-1}\right.$ $\left.\mathrm{s}^{-1}\right)$ and longer $\lambda_{\mathrm{MFL}}(1 \mu \mathrm{m}){ }^{21}$ The utilization of h-BN as the encapsulated layer provides a flat surface to the active graphene layer and eliminates the traps that generate high mobility charge carriers. In order to reduce significant cost in experiments for the optimization of the device geometry, simulations are typically being used. Recently, the simulation and modeling of graphene based TTJ rectifiers have been reported, considering a carrier mobility of $4000 \mathrm{~cm}^{2} \mathrm{~V}^{-1} \mathrm{~s}^{-1}$ to compare the simulated results with the reported data. ${ }^{11,25}$

The simulations are typically being utilized for device geometry optimization and hence reduce the significant cost of an experiment. However, no simulation effort has been made on the graphene FTBR until now. Thus in this work, a simulation study along with noise analysis of the graphene FTBR with a triangular antidot is performed and validated with the equivalent resistor network. The DC and RF characteristics are analyzed in combination with trap effects at room temperature and $\mathrm{THz}$ frequency. Previously, only two dimensional (2D) MC simulations of InGaA based nanodevices have been performed where the front and top view of the device was considered separately. ${ }^{6}$ In contrast, three dimensional (3D) simulations in addition to $2 \mathrm{D}$ simulations are reported and compared in this work, which is a more accurate approach. In addition, the high frequency (THz) operating capability of the FTBR is validated with the responsivity and noise equivalent power (NEP) characteristics. Furthermore, the low frequency behavior of the FTBR is also simulated with an equivalent $2 \mathrm{D}$ structure and is validated with the theory.

\section{Modeling of the graphene FTBR}

The FTBR as shown in Fig. 1(a) is simulated using single layer graphene with a thickness of $0.35 \mathrm{~nm}$, and a highly doped $\mathrm{Si}$ / $\mathrm{SiO}_{2}$ substrate with a dielectric thickness $(t)$ and permittivity $(\varepsilon)$ of $285 \mathrm{~nm}$ and 3.9, respectively. The models for the graphene based structure are validated with a carrier mobility $(\mu)$ of 4000 $\mathrm{cm}^{2} \mathrm{~V}^{-1} \mathrm{~s}^{-1},{ }^{25}$ which corresponds to $\lambda_{\mathrm{MFL}}=(h / 2 e) \mu\left(n_{\mathrm{s}} / \pi\right)^{1 / 2}$ of $39 \mathrm{~nm} .{ }^{24}$ Here, $h$ is the Planck's constant and $e$ is the electron charge. The carrier density for graphene is estimated with a parallel plate capacitor model using $n_{\mathrm{s}}=\varepsilon_{0} \varepsilon V_{\mathrm{G}} / t e$, where $\varepsilon_{0}$ is the free space permittivity and $V_{\mathrm{G}}$ is the back gate voltage. ${ }^{26}$ As seen from Fig. 1(a), the device active region of the proposed ballistic rectifier has an antidot (triangular structure) at the center which deflects the charge carriers either originated from the source (S) or drain (D) terminal to the lower (L) terminal. The length of the upper side wall of the antidot triangle is $30 \mathrm{~nm}$ and the available channel lengths between upper and source/ drain terminals are $35 \mathrm{~nm}$ each which is less than the mean free path length of $39 \mathrm{~nm}$. However, the overall length of the device is $250 \mathrm{~nm}$ which is larger than the mean free path length. Hence, the drift-diffusion model is utilized to compute/estimate the electrical characteristics of the device analogous to the approach utilized for evaluating the electrical characteristics of the InGaA based ballistic rectifier implementing Monte Carlo simulations. ${ }^{6,7}$ Silvaco Atlas software is used for modeling and
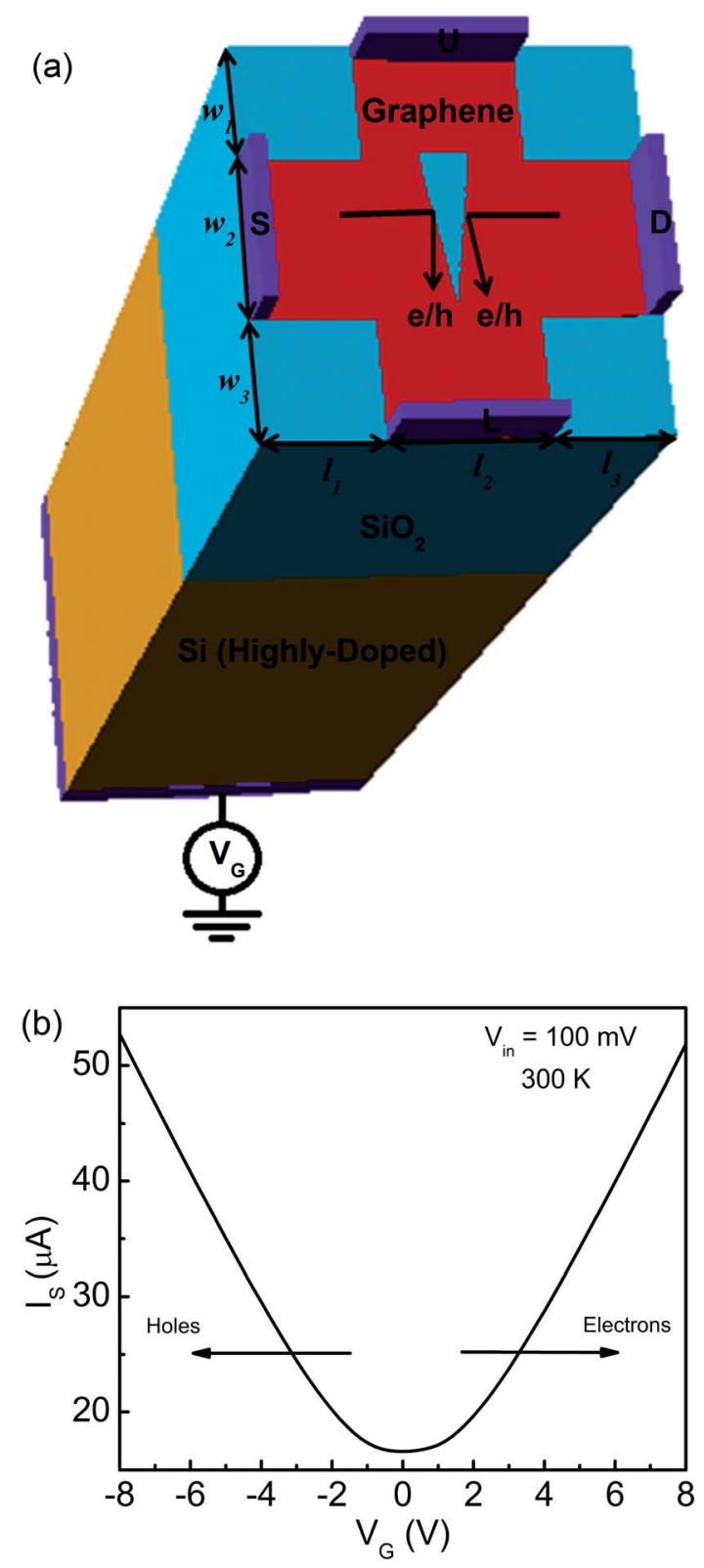

Fig. 1 (a) 3D device structure of the graphene FTBR with a highly doped $\mathrm{Si} / \mathrm{SiO}_{2}$ substrate. The triangular antidot at the centre of the device deflects a large number of electrons and/or holes toward the lower terminal as indicated by arrows. The dimensions are $l_{1}=l_{3}=w_{1}$ $=w_{3}=75 \mathrm{~nm}$ and $l_{2}=w_{2}=100 \mathrm{~nm}$. The FTBR terminals are source (S), drain (D), lower (L), and upper (U). The rectified response is generated between $L$ and $U$ terminals when a non-zero input is applied between $S$ and $D$ terminals. (b) The transfer characteristic of the FTBR shows input branch current $\left(I_{\mathrm{S}}\right)$ as a function of applied back gate voltage $\left(V_{\mathrm{G}}\right)$ for a push-pull input voltage of $100 \mathrm{mV}$ between $\mathrm{S}$ and $\mathrm{D}$ terminals in push-pull (PP) configuration.

simulation of the graphene FTBR, in which Poisson's and continuity equations are used for a general framework to corelate the electrostatic potential with the charge density. ${ }^{27}$ Also, the continuity equation is used in combination with transport equations for carrier densities considering various effects such as generation and recombination of carriers, and transport processes. 
The drift-diffusion model ${ }^{25}$ is used to calculate the current densities $J_{\mathrm{n}}$ and $J_{\mathrm{p}}$, and carrier concentrations $\mathrm{n}$ and $\mathrm{p}$ for electrons and holes, respectively, which depend on quasi-Fermi levels denoted by $\varphi_{\mathrm{n}}$ and $\varphi_{\mathrm{p}}$.

$$
\begin{gathered}
J_{\mathrm{n}}=-e \mu_{\mathrm{n}} \mathrm{n} \nabla \varphi_{\mathrm{n}} \\
J_{\mathrm{p}}=-e \mu_{\mathrm{p}} \mathrm{p} \nabla \varphi_{\mathrm{p}} \\
n=n_{\mathrm{i}} \exp \left[\frac{e\left(\psi-\phi_{\mathrm{n}}\right)}{k T}\right] \\
p=n_{\mathrm{i}} \exp \left[\frac{-e\left(\psi-\phi_{\mathrm{p}}\right)}{k T}\right]
\end{gathered}
$$

where $\mu_{\mathrm{n}}$ and $\mu_{\mathrm{p}}$ are the electron and hole mobility, respectively, $n_{\mathrm{i}}$ is the intrinsic concentration of carriers, $\psi$ is the electrostatic potential, $k$ is the Boltzmann constant and $T$ is the lattice temperature in Kelvin.

The field dependent mobility model is used to formulate the effective mobility of carriers and is given as

$$
\begin{aligned}
& \mu_{\mathrm{n}}(E)=\mu_{\mathrm{no}}\left[\frac{1}{1+\left(\frac{\mu_{\mathrm{no}} E}{\nu_{\mathrm{n}}}\right)^{\xi_{\mathrm{n}}}}\right]^{1 / \xi_{\mathrm{n}}} \\
& \mu_{\mathrm{p}}(E)=\mu_{\mathrm{po}}\left[\frac{1}{1+\left(\frac{\mu_{\mathrm{po}} E}{\nu_{\mathrm{p}}}\right)^{\xi_{\mathrm{p}}}}\right]^{1 / \xi_{\mathrm{p}}}
\end{aligned}
$$

where $E$ is the parallel electric field, $\nu_{\mathrm{n}}$ and $\nu_{\mathrm{p}}$ are the saturation velocities, and $\xi_{\mathrm{n}}$ and $\xi_{\mathrm{p}}$ are constants.

The parameters $\mu_{\text {no }}$ and $\mu_{\text {po }}$ are the low-field carrier mobilities and can be calculated using

$$
\begin{aligned}
& \mu_{\mathrm{no}}=\mu_{\mathrm{n}}\left(\frac{T}{300}\right)^{-\gamma_{\mathrm{n}}} \\
& \mu_{\mathrm{po}}=\mu_{\mathrm{p}}\left(\frac{T}{300}\right)^{-\gamma_{\mathrm{p}}}
\end{aligned}
$$

where $\mu_{\mathrm{n}}$ and $\mu_{\mathrm{p}}$ are equal to $4000 \mathrm{~cm}^{2} \mathrm{~V}^{-1} \mathrm{~s}^{-1}$ and $\gamma_{\mathrm{n}}$ and $\gamma_{\mathrm{p}}$ are constants.

The intrinsic carrier concentration can be calculated using

$$
n_{\mathrm{i}}=\sqrt{n p}=\sqrt{N_{\mathrm{c}} N_{\mathrm{v}}} \exp \left[\frac{-E_{\mathrm{g}}}{2 k T}\right]
$$

where $N_{\mathrm{c}}$ and $N_{\mathrm{v}}$ are the conduction and valence band density of states, respectively.

For single layer graphene, the band gap $\left(E_{\mathrm{g}}\right)$ is considered to be zero; therefore eqn (9) can be written as

$$
n_{\mathrm{i}}=\sqrt{n p}=\sqrt{N_{\mathrm{c}} N_{\mathrm{v}}}=N
$$

The parallel plate capacitor model is used to calculate the surface charge density of graphene which is normalized into the density of states $(N)$ using graphene thickness. It shows a similar value calculated using the Fermi integral of order 1 with intrinsic carrier concentration and is given $\operatorname{as}^{25,28}$

$$
n_{\mathrm{iF}}=\frac{2}{\pi}\left(\frac{k T}{\hbar \nu_{\mathrm{F}}}\right) F_{1}\left(\frac{E_{\mathrm{F}}}{k T}\right)
$$

where $v_{\mathrm{F}}$ is the Fermi velocity, $\hbar$ is the reduced Planck's constant, $F_{1}$ is the Fermi integral of order 1 , and $E_{\mathrm{F}}$ is the Fermi energy.

The value of $F_{1}$ cannot be integrated analytically; therefore, the following approximations are used to calculate the intrinsic carrier concentration such as $E_{\mathrm{F}}=0$ and $F_{1}=\pi^{2} / 12$ and carrier concentration becomes ${ }^{28}$

$$
n_{\mathrm{iF}}=\frac{\pi}{6}\left(\frac{k T}{\hbar v_{\mathrm{F}}}\right)^{2}
$$

The value of $n_{\mathrm{iF}}$ can be calculated for different temperatures using eqn (12).

\section{Results and discussion}

\section{Three dimensional (3D) structure and simulation}

The FTBR is designed on single layer graphene having fourterminals, source (S), drain (D), lower (L), and upper (U), as demonstrated in Fig. 1(a). A triangular antidot is intentionally placed at the center of the device to provide asymmetry between output terminals and deflect a large number of electrons and/or holes toward the L terminal as compared to the U terminal. The $\mathrm{Si} / \mathrm{SiO}_{2}$ substrate is back gated with an electrode that can tune the type of charge carriers in the graphene layer from electrons to holes and vice versa. The collimation of charge carriers inside the channel is dominating which changes the transmission probability of electrons/holes between the input (S and D) and output ( $\mathrm{L}$ and $\mathrm{U}$ ) terminals and results in the rectified output. ${ }^{20}$ The transfer characteristic of the FTBR for an input voltage of $100 \mathrm{mV}$ in push-pull (PP) configuration demonstrating the zero Dirac point considering ideal graphene is shown in Fig. 1(b), and the voltage $\left(V_{\mathrm{G}}\right)$ is applied on the back gate electrode. The left and right sides of the curve demonstrate hole and electron dominant regions, respectively.

The output response of the FTBR as a function of $V_{\mathrm{G}}$ is shown in Fig. 2(a). The traps with a charge density of $0.1 \times 10^{12} \mathrm{~cm}^{-2}$ are considered to simulate the real device like characteristics at room temperature; however at the lower temperature, the traps become frozen out and hence are not considered. ${ }^{25}$ The rectification is reduced from $0.5 \%$ to $0.35 \%$ with traps at $300 \mathrm{~K}$. Furthermore, the efficiency of the device is increased more than three times $(1.86 \%)$ at $77 \mathrm{~K}$ and more than six times $(3.17 \%)$ at 4 $\mathrm{K}$ as shown in Fig. 2 (b). At lower temperature, the $\lambda_{\mathrm{MFL}}$ and mobility of the device increase, improving the rectification efficiency. In addition, the rectification efficiency of the FTBR can further be improved by fabricating graphene with a smooth surface and edges which in turn increases the mobility of the device. ${ }^{24}$

The output response of the device is simulated for two input voltage configurations such as push-fixed (PF) and PP for 

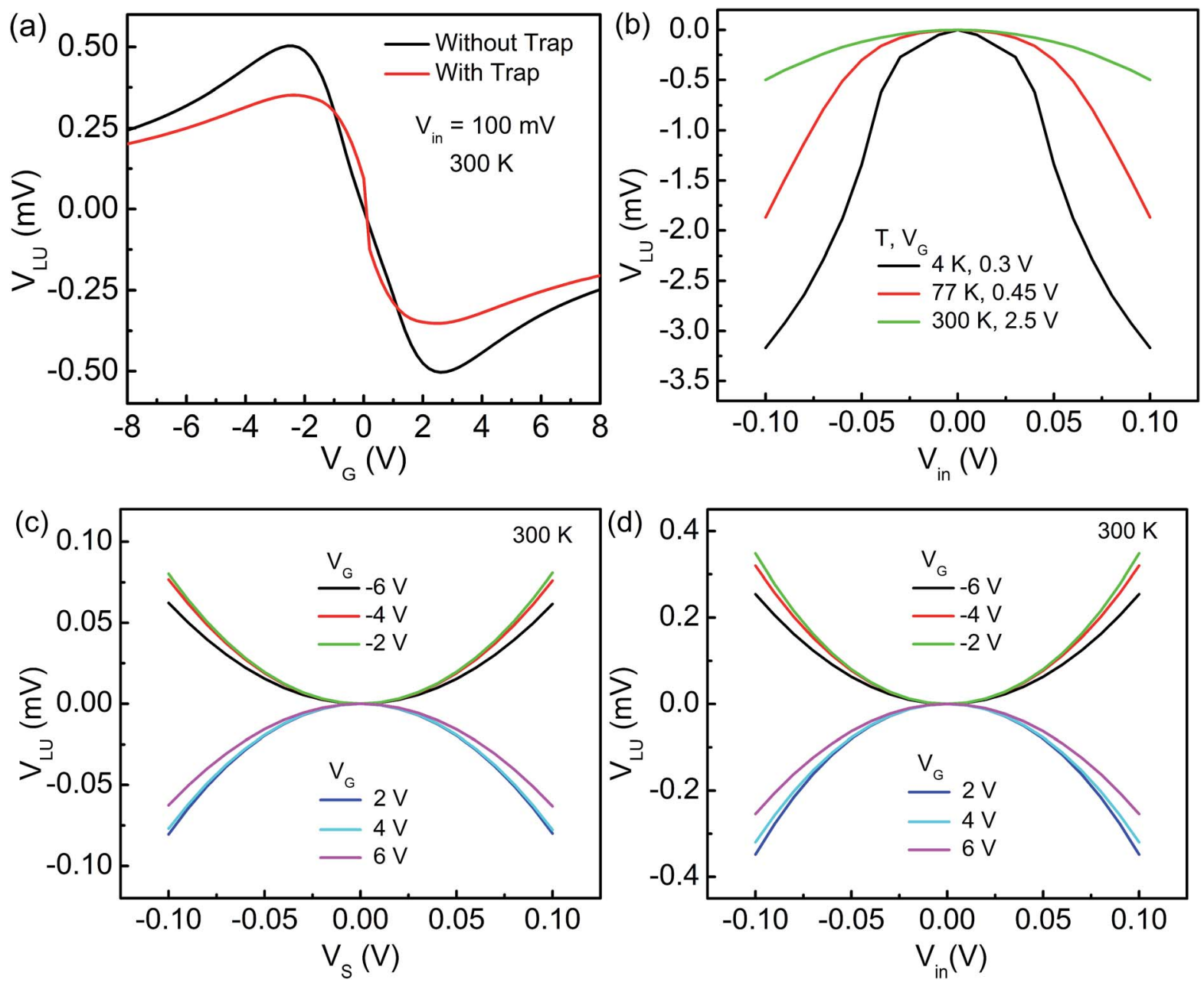

Fig. 2 (a) The output response of the FTBR with and without traps for different values of $V_{G}$. (b) Input-output response of the rectifier at different temperatures. The FTBR is biased using (c) push-fixed (PF) and (d) PP input configurations. Both the input configurations are simulated for electrons (downward) and holes (upward) at different $V_{G}$. The rectifier can be used as a voltage inverter by changing the $V_{G}$ from positive to negative and vice versa.

different $V_{\mathrm{G}}$ as shown in Fig. 2(c) and (d), respectively. The nonlinear output is related to the non-zero input as $^{29}$

$$
V_{\mathrm{LU}}=\frac{3 e}{8 \pi E_{\mathrm{F}}} \frac{N_{\mathrm{SD}} \sin 2 \theta_{\mathrm{o}}}{2 N_{\mathrm{LU}}-3 N_{\mathrm{SD}}\left(1-2 \sin \theta_{\mathrm{o}}\right)^{2}} V_{\mathrm{SD}}^{2}
$$

where $V_{\mathrm{LU}}$ is the rectified output measured in response of input voltage $\left(V_{\mathrm{SD}}\right), N_{\mathrm{SD}}$ and $N_{\mathrm{LU}}$ are the number of modes in the input and output terminals, respectively, and $\theta_{\mathrm{o}}$ is the ejection angle of charge carriers.

Eqn (13) shows the quadratic relationship between the input and output voltages and can be further reduced to $V_{\mathrm{LU}}=$ $\pm \alpha V_{\mathrm{SD}}{ }^{2}$, where $\alpha$ is the curvature of the curve ( $-\alpha$ for electrons and $+\alpha$ for holes). Both PF and PP input configurations can be extracted from each other. The output in PF input configuration at $V_{\mathrm{S}}=2 V_{\text {in }}$ and $V_{\mathrm{D}}=0$ is equal to the output in PP input configuration at $V_{\text {in }}=V_{\mathrm{S}}=-V_{\mathrm{D}}$, where $V_{\mathrm{S}}$ and $V_{\mathrm{D}}$ are the source and drain voltages, respectively, and $V_{\text {in }}$ is the PP input voltage. Both the input configurations demonstrate the rectifier function as a voltage inverter by switching the charge carriers from electrons to holes and vice versa using $V_{\mathrm{G}}$. In addition, the Fermi levels of $\mathrm{S}$ and $\mathrm{D}$ terminals are changed when the input is applied; as a result, charge carriers start flowing from one terminal to another terminal. Due to the presence of the triangular antidot, more number of charge carriers deflect toward the $\mathrm{L}$ terminal. Accordingly, the charge carrier difference between $\mathrm{L}$ and $\mathrm{U}$ terminals generates the rectified DC output $\left(V_{\mathrm{LU}}\right)$.

The AC response of the graphene FTBR in pp configuration is simulated at $1 \mathrm{KHz}$ as shown in Fig. 3(a). The DC rectified output demonstrates device operation as a frequency doubler. Furthermore, the back gate electrode affects the FTBR efficiency largely and switches the carriers for conduction. Therefore the speed of the ballistic rectifier is required to be confirmed at different $V_{\mathrm{G}}$ using responsivity and NEP characteristics as shown in Fig. 3(b) and (c), respectively. The responsivity measures the speed in terms of output voltage generated by a rectifier in response to the applied input power and NEP is the ratio of thermal voltage to the responsivity of the device. The ballistic rectifier generates the thermal noise voltage of $8.7 \mathrm{nV} \mathrm{Hz}^{-1 / 2}$ corresponding to the equivalent 

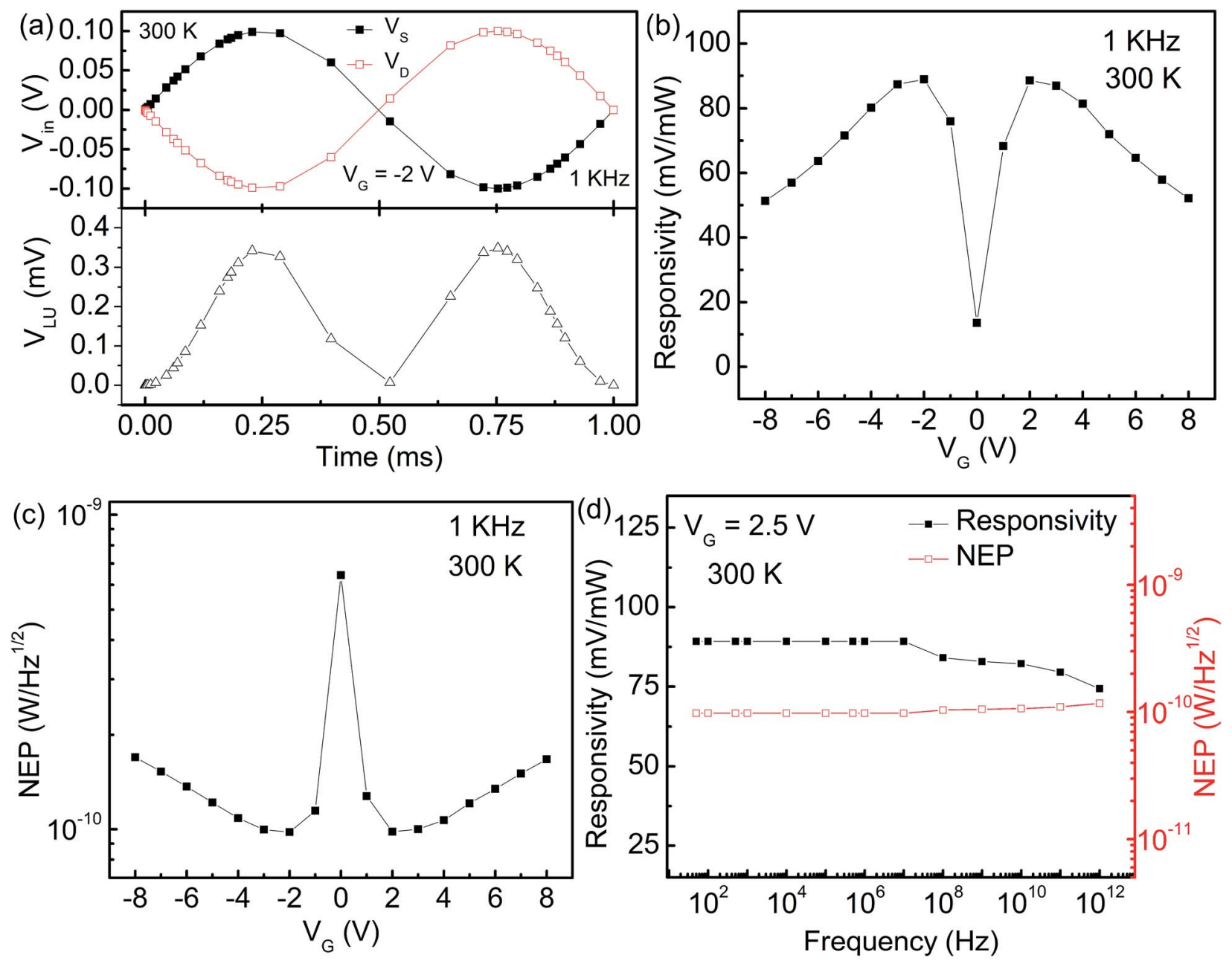

Fig. 3 (a) AC characteristics of the FTBR simulated at $1 \mathrm{KHz}$ for $V_{S}=-V_{D}=0.1 \mathrm{VPP}$ configuration demonstrate the device working as a frequency doubler. The RF performance of the rectifier is demonstrated using (b) responsivity and (c) NEP at different $V_{G}$ for electrons and holes, respectively. Similarly, the speed of the device is validated in (d) by increasing the frequency up to the $\mathrm{THz}$ range.

resistance $R_{3 \mathrm{D}}$ of $4.57 \mathrm{~K} \Omega$ calculated using $\sqrt{4 K T R_{3 \mathrm{D}}}$. The device demonstrates a responsivity of up to $88.89 \mathrm{mV} \mathrm{mW}^{-1}$ and NEP of $97.87 \mathrm{pW} \mathrm{Hz}^{-1 / 2}$ at a $V_{\mathrm{G}}$ of $-2 \mathrm{~V}$. The very first graphene based four terminal ballistic rectifier on the $\mathrm{SiO}_{2}$ substrate demonstrated a responsivity of about $66.68 \mathrm{mV} \mathrm{mW}^{-1}$ and NEP in the order of $10^{-9} \mathrm{~W} \mathrm{~Hz}^{-1 / 2}$ with a carrier mobility of 1872 $\mathrm{cm}^{2} \mathrm{~V}^{-1} \mathrm{~s}^{-1} \cdot{ }^{24}$ However, recent experiments on the graphene based FTBR utilizing the h-BN substrate exhibiting a carrier mobility of $200000 \mathrm{~cm}^{2} \mathrm{~V}^{-1} \mathrm{~s}^{-1}$ have demonstrated the significant improvement ( $>300$ times) in the responsivity of $23000 \mathrm{mV} \mathrm{mW}^{-1}$ and NEP as low as $0.64 \mathrm{pW} \mathrm{Hz}^{-1 / 2}$. $^{21}$ This further suggests that the device detection sensitivity and NEP improve with the increase in carrier mobility ensuring purely ballistic transport. In addition, it is worth mentioning that the NEP of graphene based ballistic rectifiers is comparable to that of commercially available uncooled terahertz detectors e.g. bolometers, Goley cells etc. ${ }^{24}$ These values can be further improved by optimizing the device dimensions and utilizing a substrate that provides better mobility in the graphene layer. Furthermore, the device responds well up to a frequency of 1
THz as demonstrated in Fig. 3(d). The graphene FTBR is expected to operate up to much higher frequencies by carefully designing the device with matched input-output impedance to ensure maximum power transfer between input and output terminals.

\section{Two dimensional (2D) structure and simulation}

The 2D structure of the ballistic rectifier is developed using a single layer of graphene and the results are compared with 3D simulations. Fig. 4(a) and (b) show the 2D structure of the FTBR with PF and PP input configurations, respectively. The approach required to analyze the noise behaviour of the FTBR as a 3D noise simulator is not available. Previously, the $2 \mathrm{D}$ approach was reported to simulate the III-V material ballistic device without considering the 3D geometry. ${ }^{6,7}$ Instead, here 3D and 2D geometries are simulated separately, and their results are compared, which is a more accurate approach.

In PF configuration, the input is applied either from the $\mathrm{S}$ or D terminal $\left(V_{\mathrm{S}}=2 V_{\text {in }}\right.$ and $\left.V_{\mathrm{D}}=0\right)$ at $V_{\mathrm{G}}=2.5 \mathrm{~V}$ and rectified response is measured between $\mathrm{L}$ and $\mathrm{U}$ terminals as shown in 


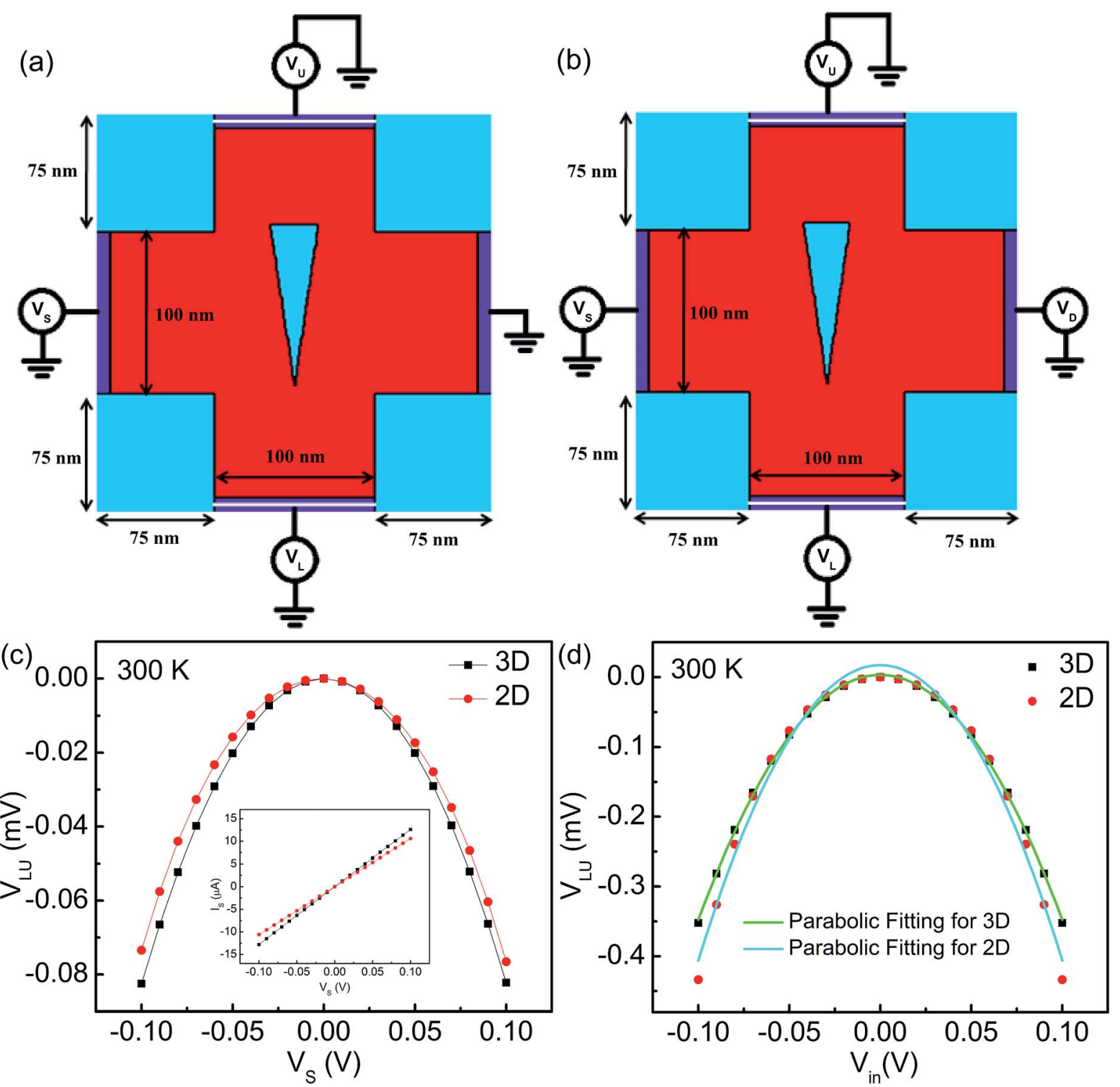

Fig. 4 Device active region is simulated in two dimensional (2D) for PF (a and c) and PP (b and d) input configurations. The input-output response of the ballistic rectifier is optimized and compared for $V_{G}=2.5 \mathrm{~V}$. The PP response of the rectifier is according to the rectified response of the device $V_{\mathrm{LU}}= \pm \alpha V_{\mathrm{SD}}{ }^{2}$, as shown by parabolic fitting.

Fig. 4(c). In PP configuration as shown in Fig. 4(d), the input is applied between $\mathrm{S}$ and $\mathrm{D}$ terminals $\left(V_{\mathrm{in}}=V_{\mathrm{S}}=-V_{\mathrm{D}}\right)$ at $V_{\mathrm{G}}=2.5 \mathrm{~V}$ and rectified response is measured between $\mathrm{L}$ and $\mathrm{U}$ terminals. The 3D and 2D responses of the ballistic rectifier are parabolic $\left(V_{\mathrm{LU}}= \pm \alpha V_{\mathrm{SD}}{ }^{2}\right)$ as given in eqn (13), where $\alpha$ is equal to -0.0351 and $-0.0423 \mathrm{~V}^{-1}$, respectively, which is calculated using quadratic curve fitting. Furthermore, the optimized 2D geometry is used to analyze the noise behaviour of the ballistic rectifier.

\section{Noise analysis of the FTBR}

The FTBR has zero intrinsic threshold which eliminates the use of a biasing circuit even at higher frequencies. In addition, output terminals in the FTBR are orthogonal to input terminals, which offer less noise as compared to the conventional $\mathrm{p}-\mathrm{n}$ diode. ${ }^{21}$ However, the analysis of low frequency noise is required as in RF design it translates into the phase noise at higher frequencies. ${ }^{30}$ The $2 \mathrm{D}$ structure of the ballistic rectifier is simulated for noise spectra from $0.1 \mathrm{~Hz}$ to $1 \mathrm{THz}$ frequency. The equivalent resistor network of the rectifier is demonstrated in Fig. 5(a), where resistance between any two terminals is calculated by taking other terminals as the open circuit represented by $R_{\mathrm{A}}, R_{\mathrm{B}}, R_{\mathrm{C}}, R_{\mathrm{D}}$ and $R_{\mathrm{E}}$. The resistor network in Fig. $5(\mathrm{~b})$ can be extracted such that $R_{\mathrm{E}}=R_{1}+R_{2}$ and $R_{\mathrm{A}}=R_{\mathrm{B}}=R_{\mathrm{C}}=R_{\mathrm{D}}=R_{1}+R_{3}=R_{2}+R_{4} \cdot{ }^{31}$ The ballistic rectifier generates a thermal noise resistance $\left(R_{2 \mathrm{D}}\right)$ of $4.88 \mathrm{~K} \Omega$ that is in good agreement with the resistor network of value $5.7 \mathrm{~K} \Omega$. Furthermore, the thermal noise $\left(\sqrt{4 K T R_{2 \mathrm{D}}}\right)$ calculated from 2D noise simulation of the FTBR is $8.99 \mathrm{nV} \mathrm{Hz}^{-1 / 2}$ which is in good accordance with a 3D thermal noise of $8.7 \mathrm{nV} \mathrm{Hz}^{-1 / 2}$. 
(a)

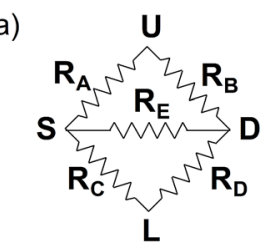

(b)

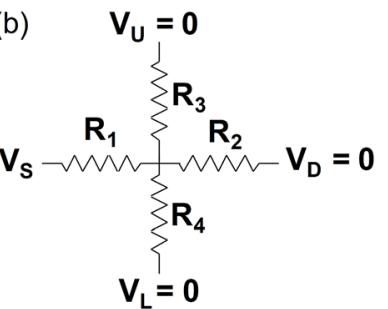

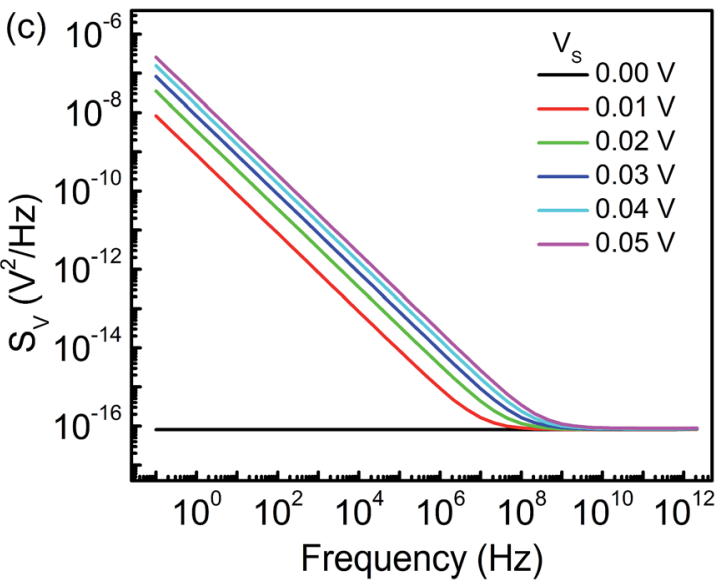

Fig. 5 ( $(a$ and b) Equivalent resistive network of the graphene FTBR. (c) Noise spectra of the ballistic rectifier are simulated from the S terminal of the device for different values of input voltages. The thermal noise is generated at $V_{\mathrm{s}}=0 \mathrm{~V}$ that matched with the resistance calculated from the resistor network. Fitting of noise spectra results in $\beta=1 \pm 0.001$ which is in analogous with the Hooge's theory.

The traps are inherently present in the structure due to the rough surface provided by the $\mathrm{SiO}_{2}$ substrate which decreases the mobility of charge carriers in the graphene layer and directly affects the number of carriers generating low frequency noise. The spectral density of low frequency noise is inversely proportional to applied signal frequency and is known as flicker
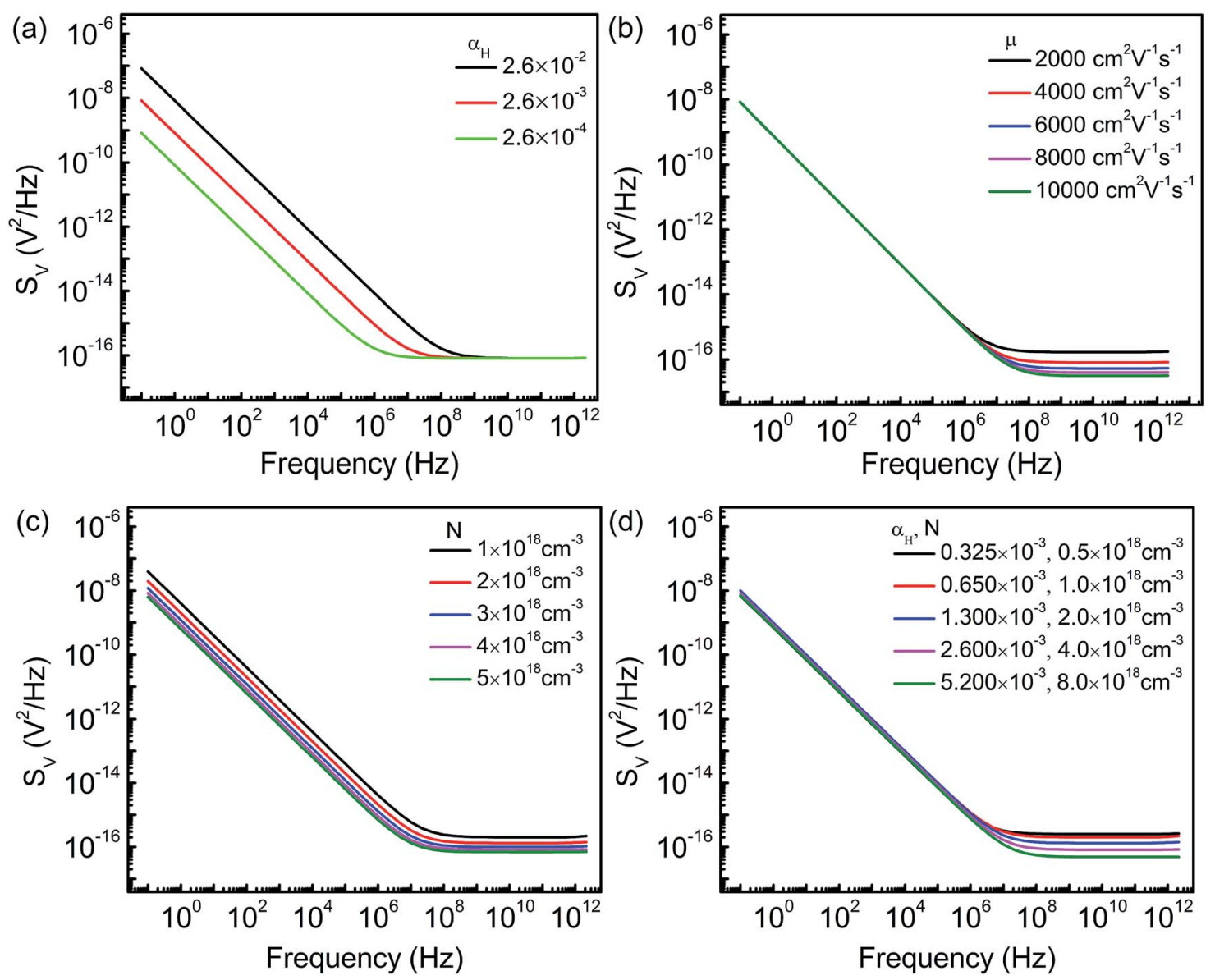

Fig. 6 (a) The simulated noise spectra of the FTBR for different $\alpha_{H}$ orders demonstrating the effect on the flicker noise $\left(\mu=4000 \mathrm{~cm}^{2} V^{-1} \mathrm{~s}^{-1} ; N^{-}\right.$ $\left.=4 \times 10^{18} \mathrm{~cm}^{-3}\right)$. (b) The mobility variations in the device channel do not affect the flicker noise, but change the thermal noise $\left(\alpha_{H}=2.6 \times 10^{-3}\right.$; $N=4 \times 10^{18} \mathrm{~cm}^{-3}$ ). (c) Both the flicker and thermal noise of the ballistic rectifier are varied with the change in the number of charge carriers in the channel $\left(\alpha_{\mathrm{H}}=2.6 \times 10^{-3} ; \mu=4000 \mathrm{~cm}^{2} \mathrm{~V}^{-1} \mathrm{~s}^{-1}\right)$. (d) Similar phenomenon is observed by changing $\alpha_{\mathrm{H}}$ and $N$ with the same amplitude, where flicker noise is not affected due to equal change in $\alpha_{\mathrm{H}}$ and $N\left(\mu=4000 \mathrm{~cm}^{2} V^{-1} \mathrm{~s}^{-1}\right)$. 
noise or $1 / f$ noise. The flicker noise can be formulated using Hooge's relation given below: ${ }^{30,32}$

$$
S_{\mathrm{V}}(f)=\frac{\alpha_{\mathrm{H}} V^{2}}{N_{\mathrm{T}} f^{\beta}}=\frac{A V^{2}}{f^{\beta}}
$$

where $S_{\mathrm{V}}(f)$ is the voltage noise spectral density, $\alpha_{\mathrm{H}}$ is the Hooge's constant, $V$ is the applied voltage, $N_{\mathrm{T}}$ is the total number of charge carriers that take part in the conduction, ${ }^{\mathbf{1 6}}$ and $\beta$ is the constant with an estimated value of $1 \pm 0.001$. The noise amplitude is equal to $A=\alpha_{\mathrm{H}} / N_{\mathrm{T}}$. The value of the Hooge's constant is considered as $2.6 \times 10^{-3}$ which is of the same order as considered for carbon nanotubes. ${ }^{30}$

The spectral density of the FTBR for different input voltages is shown in Fig. 5(c). The curve at higher voltage coincides with the thermal noise at $\mathrm{MHz}$ frequencies. Also, the noise spectra are directly proportional to the square of applied voltage according to eqn (14). In addition, the noise spectra also vary with the number of factors including the Hooge's constant $\left(\alpha_{\mathrm{H}}\right)$ and number of charge carriers $\left(N_{\mathrm{T}}\right)$. The charge carriers and mobility are related to each other as given by the conductivity $(\sigma)$ or resistivity $(\rho)$ expression as

$$
\sigma=\frac{1}{\rho}=e \mu n_{\mathrm{s}}
$$

The spectral density is simulated for different values of $\alpha_{\mathrm{H}}$ (up to three orders of magnitude) which demonstrate change in the $1 / f$ noise as shown in Fig. 6(a). The mobility variations did not affect flicker noise but suggest the change in thermal noise due to the change in device resistance as shown in Fig. 6(b).

In addition, the number of charge carriers is varied as shown in Fig. 6(c), which affects both the flicker noise and device resistance according to eqn (14) and (15). The results suggest that the flicker noise is due to the fluctuation in carrier concentration rather than mobility. The effect of carrier fluctuation on $1 / f$ noise can be minimized by utilizing a suitable material having appropriate $\alpha_{\mathrm{H}}$ as shown in Fig. 6(d).

\section{Conclusions}

The graphene FTBR is simulated using the drift-diffusion model. The obtained results for $3 \mathrm{D}$ and $2 \mathrm{D}$ geometries are compared with each other for PF and PP input configurations. The device demonstrates a responsivity and NEP of $89.21 \mathrm{mV}$ $\mathrm{mW}^{-1}$ and $97.52 \mathrm{pW} \mathrm{Hz}^{-1 / 2}$, respectively. The noise characteristics of the graphene FTBR suggest that the device exhibits low frequency noise, which is mainly due to the number of charge carriers available in the channel, and is not affected by mobility. In addition, the device speed can be enhanced by producing graphene on a suitable substrate having properties without affecting low frequency noise. Accordingly, the graphene ballistic rectifier can be used as a room temperature detector at high microwave/terahertz frequencies producing a high rectification ratio similar to a graphene self-switching device ${ }^{33}$ for various applications including $\mathrm{THz}$ imaging.

\section{Conflicts of interest}

There are no conflicts to declare.

\section{Acknowledgements}

This work was supported by the Science and Engineering Research Board (SERB), Department of Science and Technology (DST), Govt. of India (SB/S3/EECE/002/2016). AG acknowledges financial support from Punjab Engineering College (Deemed to be University), Chandigarh, India.

\section{References}

1 K. I. Bolotin, K. J. Sikes, Z. Jiang, M. Klima, G. Fudenberg, J. Hone, P. Kim and H. L. Stormer, Solid State Commun., 2008, 146, 351-355.

2 K. S. Novoselov, V. I. Fal'ko, L. Colombo, P. R. Gellert, M. G. Schwab and K. Kim, Nature, 2012, 490, 192-200.

3 M. Y. Han, B. Özyilmaz, Y. Zhang and P. Kim, Phys. Rev. Lett., 2007, 98, 206805.

4 L. Britnell, R. V. Gorbachev, R. Jalil, B. D. Belle, F. Schedin, A. Mishchenko, T. Georgiou, M. I. Katsnelson, L. Eaves, S. V. Morozov, N. M. R. Peres, J. Leist, A. K. Geim, K. S. Novoselov and L. A. Ponomarenko, Science, 2012, 335, 947-950.

5 G. Auton, R. K. Kumar, E. Hill and A. Song, J. Electron. Mater., 2017, 46, 3942-3948.

6 J. Mateos, B. G. Vasallo, D. Pardo, T. Gonzalez, J. Galloo, S. Bollaert, Y. Roelens and A. Cappy, IEEE Trans. Electron Devices, 2003, 50, 1897-1905.

7 J. Mateos, B. G. Vasallo, D. Pardo, T. González, J. S. Galloo, Y. Roelens, S. Bollaert and A. Cappy, Nanotechnology, 2003, 14, 117.

8 V. Kaushal, I. Iñiguez-de-la-Torre and M. Margala, Solid-State Electron., 2011, 56, 120-129.

9 H. Q. Xu, Appl. Phys. Lett., 2001, 78, 2064-2066.

10 A. Jacobsen, I. Shorubalko, L. Maag, U. Sennhauser and K. Ensslin, Appl. Phys. Lett., 2010, 97, 032110.

11 P. Butti, I. Shorubalko, U. Sennhauser and K. Ensslin, J. Appl. Phys., 2013, 114, 033710.

12 A. M. Song, A. Lorke, A. Kriele, J. P. Kotthaus, W. Wegscheider and M. Bichler, Phys. Rev. Lett., 1998, 80, 3831-3834.

13 A. M. Song, Encycl. Nanosci. Nanotechnol., 2004, 9, 371-389. 14 Q. Diduck, M. Margala and M. Feldman, IEEE MTT-S Int. Microw. Symp. Dig., IEEE, 2006.

15 Q. Diduck, H. Irie and M. Margala, Int. J. High Speed Electron. Syst., 2009, 19, 23-31.

16 A. K. Singh, S. R. Kasjoo and A. M. Song, IEEE Trans. Nanotechnol., 2014, 13, 527-531.

17 A. M. Song, Appl. Phys. A, 2002, 75, 229-235.

18 A. M. Song, P. Omling, L. Samuelson, W. Seifert, I. Shorubalko and H. Zirath, Appl. Phys. Lett., 2001, 79, 1357-1359.

19 D. Wolpert, Q. Diduck and P. Ampadu, IEEE Trans. Nanotechnol., 2009, 10, 150-154. 
20 A. M. Song, Phys. Rev. B: Condens. Matter Mater. Phys., 1999, 59, 9806-9809.

21 G. Auton, J. Zhang, R. K. Kumar, H. Wang, X. Zhang, Q. Wang, E. Hill and A. Song, Nat. Commun., 2016, 7, 11670.

22 A. Garg, N. Jain and A. K. Singh, IEEE Int. Conf. Recent Trends Electron. Inf. Commun. Technol., IEEE, 2016.

23 G. Auton, D. B. But, J. Zhang, E. Hill, D. Coquillat, C. Consejo, P. Nouvel, W. Knap, L. Varani, F. Teppe, J. Torres and A. Song, Nano Lett., 2017, 17, 7015-7020.

24 A. K. Singh, G. Auton, E. Hill and A. Song, Carbon, 2015, 84, 124-129.

25 A. Garg, N. Jain and A. K. Singh, J. Comput. Electron., 2018, 17, 562-570.

26 K. S. Novoselov, A. K. Geim, S. V. Morozov, D. Jiang, Y. Zhang, S. V. Dubonos, I. V. Grigorieva and A. A. Firsov, Science, 2004, 306, 666-669.
27 ATLAS user's manual-device simulation software, Silvaco, Inc., 2012.

28 H. S. P. Wong and D. Akinwande, Carbon nanotube and graphene device physics, Cambridge University Press, Cambridge, 2010.

29 A. M. Song, S. Manus, M. Streibl, A. Lorke, J. P. Kotthaus, W. Wegscheider and M. Bichler, Superlattices Microstruct., 1999, 25, 269-272.

30 Y. M. Lin and P. Avouris, Nano Lett., 2008, 8, 2119-2125.

31 I. Iñiguez-de-la-Torre, T. González, D. Pardo, C. Gardès, Y. Roelens, S. Bollaert, A. Curutchet, C. Gaquiere and J. Mateos, Semicond. Sci. Technol., 2010, 25, 125013.

32 F. N. Hooge, T. G. M. Kleinpenning and L. K. J. Vandamme, Rep. Prog. Phys., 1981, 44, 479-532.

33 A. K. Singh, G. Auton, G. Hill and A. Song, 2D Mater., 2018, 5, 035023. 